Questions de communication

\title{
Circulation, altération et appropriation d'une information scientifique
}

Quand les silures attaquent les pigeons

Circulation, Alteration and Appropriation of Scientific Information. "When

Catfish attack Pigeons"

\section{Robert Boure et Muriel Lefebvre}

\section{(2) OpenEdition}

Journals

Édition électronique

URL : http://journals.openedition.org/questionsdecommunication/8690

DOI : 10.4000/questionsdecommunication.8690

ISSN : 2259-8901

Éditeur

Presses universitaires de Lorraine

\section{Édition imprimée}

Date de publication : 31 décembre 2013

Pagination : 169-198

ISBN : 978-2-8143-0182-5

ISSN : 1633-5961

Référence électronique

Robert Boure et Muriel Lefebvre, « Circulation, altération et appropriation d'une information scientifique », Questions de communication [En ligne], 24 | 2013, mis en ligne le 01 février 2016, consulté le 04 mai 2019. URL : http://journals.openedition.org/questionsdecommunication/8690 ; DOI : 10.4000/questionsdecommunication.8690 
ROBERT BOURE

Laboratoire d'études et de recherches appliquées en sciences sociales

Université Toulouse 3-Paul Sabatier

F-31077

robert.boure@iut-tlse3.fr

MURIEL LEFEBVRE

Laboratoire d'études et de recherches appliquées en sciences sociales

Université Toulouse 1 Capitole

F-31077

Muriel.Lefebvre@ut-capitole.fr

\section{CIRCULATION, ALTÉRATION ET APPROPRIATION D'UNE INFORMATION SCIENTIFIQUE. QUAND LES SILURES ATTAQUENT LES PIGEONS}

Résumé. - L'article tente de comprendre comment, pourquoi et avec quelles conséquences une publication de chercheurs français - mise en ligne en décembre 2012 par la revue scientifique électronique américaine PLOS ONE - est l'objet sur l'internet de ce que le langage courant désigne par le terme « buzz », alors que la circulation de littérature scientifique ne dépasse guère le champ académique. Sur le plan théorique, il s'inscrit dans une approche fondée sur la mise en perspective des travaux d'Yves Jeanneret (2008) relatifs à la « circulation des êtres culturels » hors de leur sphère d'origine, des recherches sur les pratiques sociales et discursives sur l'internet et, enfin, des travaux sur la vulgarisation scientifique, notamment ceux traitant des médiations.

Mots clés. - Articles scientifiques, revues, PLOS ONE, internet, circulation, altérations, appropriations, médiations, usages. 
L

e 5 décembre 20I2, la revue scientifique internationale de référence en ligne et en libre accès PLOS ONE publie l'article d'une équipe de chercheurs toulousains (Coucherousset et al., 2012)' révélant, d'une part, que des silures introduits dans le Tarn - rivière du Sud de la France - attaquent des pigeons sur les berges en s'échouant selon la technique du beaching utilisée par d'autres espèces et, d'autre part, que le pigeon est devenu l'élément essentiel du régime alimentaire de certains silures. Aussitôt, « la Toile s'enflamme »- selon l'expression désormais médiatiquement consacrée - au-delà du seul champ scientifique, au grand étonnement de l'équipe de recherche², peu habituée à ce phénomène que d'aucuns nomment « buzz 》 ou « emballement médiatique ». Car il est peu fréquent qu'un fait scientifique soit l'objet d'une telle exposition ${ }^{3}$, phénomène qui affecte surtout - tantôt brièvement, tantôt sur des périodes plus longues - des événements mondains, des affaires politico-judiciaires, des crises sanitaires, des cataclysmes ou, plus trivialement, des vidéo-gags mettant en scène des animaux ou des humains (le Harlem Shake, par exemple). Et lorsque la science est concernée, c'est souvent pour un événement marquant ou présumé tel sur le moment - comme une controverse internationale (les organismes génétiquement modifiés, etc.), une découverte scientifique majeure (virus du SIDA, boson de Higgs, etc.) ou renvoyant aux standards de la science-fiction (planète aux quatre soleils, exoplanète, etc.), une théorie fascinante (Big Bang, etc.).

Si le comportement de prédation des silures, révélateur d'une adaptation à un nouvel environnement, n'avait pas encore été scientifiquement observé et analysé chez cette espèce vivant en eau douce, il ne s'agit - de l'aveu même des chercheurs (entretiens) - ni d'une découverte « prometteuse » sur les plans économique, sanitaire ou environnemental, ni même de la mise en évidence d'un comportement animal inédit puisqu'on sait que les orques attrapent ainsi des phoques avant de les dévorer dans l'océan. Bref, a priori, il n'y a pas de quoi intéresser et faire réagir des publics larges, souvent très éloignés de la sphère scientifique.

\footnotetext{
' L'équipe rassemble des chercheurs relevant du Laboratoire évolution et diversité biologique (CNRS, Université Toulouse 3-Paul Sabatier, École nationale de formation agronomique) et du Laboratoire d'écologie fonctionnelle et environnement (CNRS, Université Toulouse 3-Paul Sabatier, Institut national polytechnique de Toulouse, Institut national de la recherche agronomique).

${ }^{2}$ L'origine de notre article est une demande des auteurs de la publication (Coucherousset et al., 2012) adressée au Laboratoire d'études et de recherches appliquées en sciences sociales (LERASS) en décembre 20 I2. Ils souhaitaient comprendre une situation pour eux inédite et qu'ils n'avaient pas concrètement provoquée puisque leurs contacts avec les médias furent postérieurs au « buzz » (entretiens). Preuve de leur motivation, ils ont mis à notre disposition : d'une part, une base de données préliminaire portant sur 133 sites et blogs internationaux (dont 6 I français) ayant relaté la publication et répertoriant pour chacun la date de publication, le pays, l'auteur, la source, l'éventuel contact avec l'équipe de recherche et l'URL ; d'autre part, une première liste des vidéos mises en ligne surYoutube et Dailymotion. Cette recherche a été réalisée dans le cadre d'un projet ANR portant la référence : RESOCIT projet ANR- I I-BSH I-00 I 3 .

${ }^{3}$ Si un fait scientifique et peu fréquemment l'objet d'une telle exposition, cela n'est pas non plus exceptionnel. Ainsi, et pour s'en tenir à la seule revue PLOS ONE, le I 2 septembre 20 I2, la publication d'un article sur la découverte du singe lesula provoque-t-elle un phénomène de même type et de plus vaste ampleur (voir infra).
} 
Dès lors, il est tentant de s'interroger sur la nature, l'ampleur et les formes de ce phénomène social et discursif, d'autant plus pour le chercheur en sciences de l'information et de la communication (SIC) que ce questionnement sollicite trois champs qui, dans le meilleur des cas, se croisent plutôt deux à deux : la circulation et la réception des faits et des informations scientifiques, le traitement médiatique de l'information (cadrage, mise en récit, construction des événements) et le rôle de l'internet dans la construction sociale de phénomènes, ce qui va au-delà de la sociologie des usages de l'internet. Néanmoins, s'il est indispensable de prendre ce fait médiatique au sérieux à travers son évaluation quantitative et qualitative (quelle est son ampleur et sa durée ? Comment s'est-il diffusé et avec quels effets ?), est-il pour autant nécessaire de faire appel à la notion pour le moins floue, impressionniste et souvent moralisatrice d'« emballement médiatique » pour le qualifier et le comprendre? Ne vaut-il pas mieux s'orienter vers, d'une part, des travaux qui interrogent les pratiques en ligne des journalistes ainsi que les processus plus généraux de construction médiatique des informations sur l'internet ${ }^{4}$ et, d'autre part, des objets plus délimités? Parmi ces derniers, nous citerons :

I. la rumeur (Allport, Postman, 1947 ; Morin, 1969 ; Froissart, 2002 ; Aldrin, 2005), phénomène social présentant - par-delà les positions théoriques divergentes de ceux qui l'ont étudié - quelques similitudes et cousinages (amplification, déformation du message initial, rôle des médias) avec notre objet tout en s'en écartant sur d'autres points (certitude du message initial et de sa source) ;

2. les processus de médiation qui mettent en lumière les pratiques d'acteurs sociaux que l'on désigne sous les vocables de « leaders d'opinion », « relais » ou « médiateurs »;

3. les espaces sociaux et les dispositifs dédiés à la « communication scientifique » (Kaufmann, 1993 ; Cheveigné, 1997 ; Jacobi, 1999), à la « publicisation de la science » (Pailliart, 2005) et à la « médiatisation de la science » (Fayard, 1988 ; Babou, 2004 ; Charaudeau, 2008), pour reprendre des expressions largement usitées et qui ont en commun de poser les questions de la réception, du public comme construction savante et métaphore de la société ainsi que de l'inscription du discours de et sur la science dans le débat public, y compris dans sa dimension médiatique.

Sur le plan méthodologique, cette démarche revient à étudier le phénomène in situ de façon à faire ressortir sa dimension sociale et, plus précisément, ce qu'il dit du lien social, des discours, des représentations, des échanges et des sociabilités travaillés par les rapports sociaux et le dispositif sociotechnique de l'internet. De manière plus précise, il s'agira de s'inscrire plutôt dans la perspective développée par Yves Jeanneret (2008) de la circulation médiatique des « êtres culturels », composites d'idées, d'objets et de représentations (ici un fait scientifique devenu « information ») qui se transforment « en cheminant dans les carrefours de la vie sociale » (ici les dispositifs sociotechniques de l'internet) suscitant au passage,

\footnotetext{
${ }^{4}$ Amandine Degand et Benoît Grevisse (2012 : 329-352) donnent une bibliographie permettant d'aborder ces questions dans ses multiples dimensions et par plusieurs approches.
} 
pratiques et discours créatifs d'acteurs multiples et hétérogènes permettant leur appropriation. Elle sera prolongée sur des points spécifiques (le journalisme en ligne, l'économie des médias, la pratiques des internautes) par des approches sociologiques et socio-économiques.

Sur le plan empirique, la recherche combine deux techniques. La première est celle de l'entretien avec ceux réalisés (les 13 janvier et 22 février 2013) auprès de Frédéric Santoul, porte-parole - notamment pour les médias $^{5}$ - des chercheurs toulousains. La seconde est l'analyse d'un corpus multimodal comprenant deux types de documents, c'est-à-dire 123 sites et blogs français ayant relayé et, pour certains, analysé, interprété et parfois complété l'information à l'aide de documents étrangers à l'article initial (par exemple, des photos et vidéos) et les commentaires des internautes sur ces sites et blogs. Cette liste a été établie à partir des référencements opérés par Google à partir de l'expression « silures et pigeons » dans les 25 premières pages ( I I résultats pas page) le 13 janvier $2013^{6}$. Le deuxième type de documents est représenté par la vidéo annexée à l'article de PLOS ONE ainsi que des douze autres mises en ligne depuis la France (liste établie à la même date).

\section{Une amplification et une altération rapides}

Parmi ceux qui veulent dépasser les définitions moralisatrices de l'ainsi nommé « emballement », certains ont largement recours à la mesure et à son image d'objectivité. Or, comme toute représentation, un indicateur, un baromètre, n'est pas la réalité, mais une construction de celle-ci de sorte que « le seul moyen d'en [l'emballement] prouver l'existence est d'appareiller le regard et de lui trouver une mesure » (Froissart, 201 I : |47). Nous ne considérerons donc pas les outils de mesure comme le seul ou le principal « appareil » pertinent ${ }^{7} .$. mais ne leur dénieront pas non plus tout intérêt.

\footnotetext{
5 Notre article exploite surtout les éléments d'information factuelle recueillis lors des entretiens. Les dimensions plus analytiques (discours du chercheur sur les pratiques scientifiques et de communication ou sur les valeurs et l'éthique, représentations...) seront davantage mobilisées dans des travaux ultérieurs.

${ }^{6}$ Nous sommes conscients que cette liste s'appuie sur les artefacts de Google, notamment sur les algorithmes du pagerank qui hiérarchisent les sites en fonction de leur notoriété mesurée au nombre de liens et de citations (Réseaux, 20/3). Non seulement la prudence s'imposera quand il s'agira d'interpréter les résultats, mais il sera surtout indispensable de développer des approches qualitatives.

7 Parmi ces « appareils » pertinents, citons, par exemple : l'unité de bruit médiatique (UBM) - définie par Kantar Media et Médiamétrie - qui mesure la présence médiatique d'un sujet sur une période donnée en multipliant l'audience telle que calculée par Médiamétrie par le volume (en fait l'espace rédactionnel : une page internet, une minute d'un reportage vidéo, etc.) ; I'unité de visibilité internet (UVI) de l'Institut Trendy Buzz destinée à mesurer la visibilité d'une personnalité, d'un thème ou d'une marque - il est calculé selon le nombre de citations et l'influence des sources émettrices (un panel de I 200000 sources a été constitué), le tout rapporté au volume de données indexé au cours d'une journée.
} 
En effet, le phénomène parle par son nombre et son nombre fait parler ceux qui le font circuler et/ou le commentent. Le nombre de sites et blogs ayant relayé l'article de Julien Coucherousset et de ses collaborateurs (2012) ainsi que celui de leurs visiteurs et commentateurs est donc à considérer pleinement, ce qui suppose qu'on ne le surestime, ni ne le sous-estime. II est un élément d'appréciation - parmi d'autres - d'un phénomène dans la construction duquel le nombre et l'usage du nombre jouent un rôle certain. Car si seule une poignée de sites avaient relayé l'information scientifique, notre recherche n'aurait eu aucune raison d'être entreprise. Un détour partiel par la métrologie (listes, décomptes, classements...) est donc justifié. Simultanément, le nombre est ambigu. En effet, s'il est bien un élément de preuve, de visibilité et de comparaison, il ne doit pas faire l'objet d'une lecture naïve s'en tenant au seul score. Seul compte l'ordre de grandeur qu'il exprime autant en lui-même que par rapport à d'autres faits de même nature. De sorte qu'il est pertinent de raisonner en termes d'échelles et de seuils à partir desquels les écarts laissent entrevoir des variations qui ne sont pas seulement d'ordre quantitatif. En outre, s'il prétend mesurer une audience, un public en tant qu'entité évidemment quantifiable, il atteste du travail collectif qui a été réalisé pour le construire, sans pour autant éclairer à lui seul ni la dimension sociale de ce travail, ni l'épaisseur sociale de l'objet observé. II ne peut donc sui generis rendre compte du social, ni dans sa globalité, ni dans son « infini chatoiement » (Vatin, 2010). C'est pourquoi nous avons aussi développé un travail analytique à partir de méthodes qualitatives.

\section{Une amplification remarquable?}

Si l'on s'en tient, dans un premier temps, à une approche plutôt quantitative appuyée sur un corpus que l'on cherche à faire parler sans s'appuyer uniquement sur des algorithmes et des robots, le constat est nuancé : si la circulation de cette information connaît une ampleur sortant de l'ordinaire de la diffusion des informations scientifiques, preuve qu'un seuil a été franchi, elle est très largement cantonnée à la sphère internet et, plus précisément, à certaines régions de cette dernière. Aussi les acteurs qui la relaient ne sont-ils pas seulement nombreux : ils sont hétérogènes, tant du point de vue de leur statut que de celui de leurs pratiques éditoriales.

\section{Une circulation large et accélérée}

Dés sa mise en ligne par Plos ONE le 5 décembre 2012, l'article fait l'objet d'un nombre important de visites: alors que le seuil de 8000 vues n'est atteint que par un petit nombre d'articles, le 13 janvier 2013 celui-ci est consulté plus de 52000 fois et téléchargé plus de | 300 fois, ce qui n'est toutefois pas un record. Ainsi l'article relatif à la découverte du singe lesula - publié trois mois 
auparavant - est-il « cliqué » plus de 105000 fois et téléchargé plus de 2500 fois à la même date (rubrique « Metrics » de PLOS ONE). En réalité, depuis la création de la revue (2006), il est en 96 position (sur plus de 77000 articles), et en dixième si l'on prend en compte les six derniers mois ( 15000 articles). Paradoxalement, il ne suscite que trois commentaires sur le site de la revue (contre deux pour l'étude sur le lesula). En outre, la vidéo des chercheurs mise en ligne par Channel Plosone ${ }^{8}$ reçoit en quelques jours plus d'un million de visites alors que la vidéo la plus regardée n'avait jusqu'ici été vue que par quelques 15000 visiteurs.

En France, il est pointé, cité, décrit, déconstruit et commenté par un nombre significatif de sites et de blogs dont l'analyse constituera une première photographie de la circulation, puis de l'altération de l'information. D'autant qu'il est, d'une part, repris par une dépêche de l'Agence France-Presse et, d'autre part, massivement relayé par de nombreux moteurs de recherche et agrégateurs d'informations généralistes (Google actualités, Yahoo actualités, actualités Free, Actu Orange, INNOOO, Voila...) ou spécialisés (Alvinet, Trop geek...). De façon significative, une recherche conduite sur Google France le 20 décembre 2012 à partir de l'expression « silures et pigeons » a obtenu plus de 90000 résultats (contre plus de 12000000 pour l'article sur le lesula). Ce sont donc plusieurs milliers de sites de médias et d'institutions qui publient l'information. À ces derniers, il convient d'ajouter les sites et blogs personnels, les chats, les pages individuelles, les messages postés sur les réseaux sociaux (Facebook, Twitter...) accessibles seulement à ceux qui intègrent le réseau de l'auteur, les courriels et leurs listes de diffusion et, enfin, les sMS envoyés depuis les téléphones portables, autrement dit des éléments difficiles ou impossibles à mesurer, en tout cas avec nos moyens. On notera que, selon la rubrique « Metrics » de PLOS ONE, cet article fait l'objet d'un partage à l'échelle internationale sur les réseaux sociaux à près de 500 reprises, dont plus de 400 pour Facebook. Pour s'en tenir au corpus, 123 sites et blogs publient l'information sous la forme d'un texte court ou d'un véritable article, soit 26 médias en ligne, 38 médias natifs du web (appelés aussi pure players), 17 blogs $^{9}$ et 42 sites d'organisations (associations, administrations...). Très souvent, la vidéo issue de PLOS ONE est jointe ou pointée par un lien, et régulièrement accompagnée de photographies, souvent tirées de PLOS ONE Ou de saisies d'écran de la vidéo. Par ailleurs, 59,3\% des articles donnent lieu à des commentaires d'internautes, $59 \%$ se situant dans la fourchette d'un à neuf commentaires, $41 \%$ au-dessus (dont un à $1 \mathrm{I}$ ) ${ }^{10}$.

\footnotetext{
${ }^{8}$ Accès : http://www.youtube.com/watch?v=g ly7ASI3ZkQ. Consulté le I3/0 I//3.

9 Si les blogs sont moins représentés, cela tient essentiellement au mode de référencement de Google qui les défavorise par rapport aux sites des médias et des grandes organisations.

10 Si cette information est davantage commentée que la plupart des informations scientifiques, il convient de souligner que le nombre de « posts » reste modeste par rapport à d'autres produits. Ainsi un seul article sur un match de football ou de rugby peut générer en quelques heures plus d'un millier de commentaires.
} 
Simultanément, la vidéo accompagnant l'article de PLOS ONE - quelquefois intégrée partiellement à un reportage réalisé parTéléToulouse, France 2 ou France 3 - est mise en ligne douze fois depuis la France (onze surYoutube et une sur Dailymotion). Le 25 janvier 2013, ces documents audiovisuels ont été visionnés 643870 fois, dont 609189 (95,3 \% des visites) pour celui mis en ligne par Pierre Barthélémy, journaliste scientifique du Monde et blogueur scientifique"l. Les 23 vidéos (en anglais) ont été regardées 3603072 fois et la vidéo allemande 356 fois, soit, toute langue confondue, 4247298 visites. Entre le 6 et le 12 décembre 20 I 2, I I 2 sites sur 12 I relaient l'information. Si l'on traçait une courbe, elle prendrait la forme d'une cloche (montée en puissance et décélération fortes et rapides). Dans le même ordre d'idées, on relèvera que $85 \%$ des commentaires des internautes sur les sites sont rédigés avant le 13 décembre 20 I2. La situation est comparable non seulement sur Youtube et Dailymotion ( 8 vidéos sur 12 sont mises en ligne entre le 6 et le 12 décembre 2012 et $75 \%$ des consultations sont réalisées les premiers jours), mais aussi pour l'article de PLOS ONE (80\% des visites sont effectuées avant le 13 décembre) et la vidéo de Channel Plosone.

\section{Une circulation limitée à certaines régions de l'internet}

Spécialisés dans les sciences et/ou les techniques ou non, les médias traditionnels ignorent largement l'article. En raison de leur proximité avec le lieu (le Tarn) et l'équipe de chercheurs, et à l'exception d'un journal télévisé de France 2 et du Zapping de Canal +, seuls quelques médias midi-pyrénéens, publient l'information (Télé Toulouse, France 3 Midi-Pyrénées, La Dépêche du Midi) et la traitent en partie comme une information locale. Les versions en ligne de ces médias sont plus prolixes puisque 26 sites lui consacrent un article, de tailles et de contenus très variables d'ailleurs... quand d'autres, et non des moindres, restent muets. En utilisant la fonction « recherche » ou « archives » désormais présente sur chaque site de média en ligne, nous avons dressé la longue liste de ceux qui ignorent l'information dans les deux mois qui suivent sa parution :

- chaines télévisées nationales ayant un journal télévisé et/ou des émissions scientifiques : cinq sur neuf (TFI, Arte, France 5, I-Télé, M6) ;

- presse quotidienne nationale : cinq sur dix (Aujourd'hui en France, La Croix, Les Échos, Le Figaro, L'Humanité) ;

- presse quotidienne régionale (PQR) : 19 sur 23 pour les titres dont la diffusion payée est supérieure à 50000 exemplaires par jour ${ }^{12}$ et 47 sur 53 pour l'ensemble de la PQR ;

\footnotetext{
"Le 9 décembre 2012, Nbekblog signale que la vidéo de P. Barthélémy a déjà été visionnée plus de 230000 fois.

12 II s'agit de L'Alsace, Le Courrier de l'Ouest, Le Courrier picard, Dernières Nouvelles d'Alsace, L'Est républicain, Le Journal de Saône et Loire, La Montagne, Nice Matin, La Nouvelle République du Centre-
} 
- presse magazine nationale d'actualités : dix sur onze (Courrier international, L'Express, Le Figaro magazine, Marianne, Le Nouvel Observateur, Paris Match, Pèlerin, Valeurs actuelles, La Vie, vSD) ;

- presse magazine dédiée aux sciences : quatre sur cinq (Ça m'intéresse, La Recherche, Pour la science, Science et Vie).

De fait, ce sont surtout les médias natifs de l'internet, les blogs et les sites d'organisations qui relaient l'information. Parmi les premiers, on note quelques pure players midi-pyrénéens tels Carré d'info, Millau Live et Toulouse 7. Les médias nationaux les plus importants sont aussi impliqués, qu'ils soient dédiés à l'information générale (il ne manque guère que Mediapart) ou spécialisée : par exemple, Buzzmoiça, Divertissons-nous, Gamaniak, Mort de Rire et Koreus (divertissement) ; Futura-Sciences, Le Journal de la science, Maxisciences, Techno science (sciences et techniques) ; Actuzz, Trop geek, Web et Tech (internet) ; Esoxiste, Naturellement pêche et Pêcheur (pêche). Ces 38 médias se répartissent dans les catégories suivantes : information générale ou locale (18), divertissement (cinq), sciences et techniques (quatre), pêche (trois), high tech et internet (trois), mer (un), nature (un), cinéma (un), santé (un), femmes (un). S'ils sont dispersés dans dix catégories, trois groupes concentrent $71 \%$ des sites ${ }^{13}$.

Les 17 blogs se distribuent en six catégories, les trois premières concentrant 70,5 \% du total : pêche (cinq), information générale (quatre), insolite (trois), sciences et techniques (deux), univers personnel (deux), femmes (un). Enfin, les 42 sites d'organisations se répartissent en onze catégories, mais de façon déséquilibrée, les deux premières réunissant 59,5\% des sites, et les cinq premières 80,9 \% : pêche (18), animaux (sept), nature (trois), sciences et techniques (trois), information générale ou locale (trois), divertissement (deux), commerce (deux), insolite (un), chasse (un), photographie (un), monde rural (un). Pour les 123 sites et blogs, si l'on considère qu'il s'agit initialement de la publication d'une information scientifique, on est conduit à un constat étonnant : d'une part, une dispersion des sites dans 19 catégories différentes - preuve d'une hétérogénéité, source de polyphonie -, certaines inattendues et/ou éloignées de la science ; d'autre part, une forte concentration des sites dans cinq catégories $(79,6 \%)$ au sein desquelles « information générale, locale ou pratique » (48) et « pêche » (27) sont surreprésentées, très loin devant « sciences et techniques » (neuf), « animaux » (sept) et « divertissement » (sept).

Ouest, Ouest France, Paris Normandie, Le Progrès, La Provence, Sud Ouest, Le Télégramme, L'UnionL'Ardennais, Var Matin et La Voix du Nord.

${ }^{13}$ La dispersion thématique est moins importante pour les versions en ligne des médias traditionnels et la concentration s'opère au profit des médias d'information générale ou locale, ce qui est logique compte tenu du rôle central qu'ils assurent dans le traitement et la circulation d'informations de toute nature : information générale, locale ou pratique (22), sciences et techniques (un), pêche (un), cinéma (un), étudiants (un) et enfants (un). 


\section{Polyphonie et altération}

En termes d'énoncés et d'énonciations, que se passe t-il quand la littérature profane relaie la littérature scientifique telle qu'elle s'exprime à travers ses produits les plus légitimes, les articles publiés dans les revues académiques? La réponse est-elle fondamentalement différente selon que l'on se situe peu ou prou sur le plan de la vulgarisation scientifique ou dans une perspective qui n'a pas grand chose à voir avec elle?

Parce qu'elle relève de logiques scientifiques, économiques, informationnelles, médiatiques (Cheveigné, 1997 ; Babou, 2004) et qu'elle s'adresse à plusieurs types de publics (Jacobi, 1999) dont elle a une représentation a priori ou étayée par des études, la vulgarisation scientifique traduit, tout en les interprétant, les travaux de recherche afin non seulement de les rendre accessibles par le langage et l'effort de contextualisation, mais aussi d'accrocher les non-spécialistes, de les faire réfléchir, de les sensibiliser aux enjeux scientifiques, éthiques, économiques ou politiques et, parfois, à leurs limites. Elle n'est pas une reproduction simplifiée, mais une médiation qui propose du sens à travers ses interprétations, ses approches transversales et l'intertextualité qu'elle convoque. Elle traduit, réorganise et recompose en fonction d'éléments qui relèvent des savoirs et savoir-faire (compétences professionnelles) de chaque auteur, de sa subjectivité, de ses valeurs, mais aussi d'une énonciation éditoriale qui a ses objectifs, ses logiques et ses contraintes. Elle ouvre à son tour la porte à une palette d'interprétations par ses publics et donc à d'autres « altérations », au sens exprimé parYves Jeanneret (2008). Qu'elles soient le fait des commentateurs scientifiques ou de leurs publics, ces dernières ne sont pas des «trahisons » du contenu scientifique, mais des appropriations discursives liées aux positions, objectifs et enjeux extrascientifiques de leurs auteurs et manifestant l'autonomie de ces derniers (Veron, 1985 ; Babou, 2004). Finalement, on est bien en présence de cette circulation créative dont traite Yves Jeanneret (2008), source d'altérations inévitables car structurelles.

Publié sur le blog du Monde Passeur de sciences dès le lendemain de la mise en ligne de l'article des chercheurs toulousains (Coucherousset et al., 20 I2), puis cité, pointé, commenté et, quelquefois, partiellement reproduit par plus de la moitié des sites et blogs, l'article de Pierre Barthélémy (20 I2) s'inscrit incontestablement dans le registre de la vulgarisation scientifique. En témoignent, d'une part, d'un souci réel d'identifier avec précision le travail de recherche (lien PLOS ONE, contact avec l'équipe, présentation précise de celle-ci...), d'en rendre compte (méthodologie, résultats) et, enfin, d'expliciter ses enjeux scientifiques ; d'autre part, d'une ouverture sur d'autres horizons, notamment perceptible par le vocabulaire employé (expressions toutes faites, clichés) $)^{14}$ : certaines espèces de requins « mettent 》 des volatiles « à leur menu »; les pigeons font un «brin de toilette »; les silures imitant « sans le savoir » des orques ; 15 attaques sont « couronnées de succès »; les silures se mettent à chasser « à la tombée du soleil » (ibid.).

\footnotetext{
14 On notera que le recours aux stéréotypes et aux figures de style peut aussi être le fait des chercheurs eux-mêmes dans leurs propres articles. Igor Babou et Joëlle Le Marec (2008) y voient l'interpénétration croissante des champs scientifique et communicationnel.
} 
Une quinzaine d'articles (cNRS, Université Toulouse 3-Paul Sabatier, Sciences et Avenir, Hydrobioloblog, Maxisciences, Slate, Guru méditations, Les Postiers...) sont de même nature. Mais tous les autres s'écartent plus ou moins fortement de ce registre tant du point de vue du contenu que de celui du ton ou de l'usage du matériau linguistique (abondance de poncifs, de stéréotypes, d'expressions toutes faites, références appuyées à des séries télévisées, à des personnalités de la politique ou des arts, à des événements non scientifiques). De fait, cela revient moins à remettre - consciemment ou inconsciemment - en cause une parole d'autorité scientifique, qu'à produire un discours relativement autonome sur ou à partir de la science car l'endroit d'où on parle est éloigné du champ scientifique. Le format de cet article rendant impossible une analyse précise des énoncés et énonciations, nous mettrons en évidence l'altération - au sens défini supra - à travers trois indices : le rubricage, les titres et les commentaires.

D'abord, l'altération peut être constatée à partir des rubriques au sein desquelles chacun a placé son article, sachant que si elle est systématique dans les médias, l'organisation en rubriques l'est moins pour les sites d'organisations et, surtout, pour les blogs. En outre, on notera que la plupart des sites d'associations, et notamment celles de pêcheurs, ne sont accessibles qu'aux abonnés, le non-abonné étant renvoyé au seul forum. Si l'on met de côté les forums (27 recensés) qui ne constituent pas à proprement parler des rubriques, les 76 articles « rubriqués » sont répartis dans les catégories suivantes : insolite (18); nature, environnement, écologie, planète ${ }^{15}$ (seize) ; sciences et techniques (onze) ; fun, buzz (six) ; animaux (cinq) ; actualités (cinq); Albi, Tarn (cinq) ; pêche (deux) ; zapping (deux) ; vidéos (deux) ; santé (deux), société (un) ; web (un) ; prédation (un). La dimension scientifique de l'article de Plos ONE (Coucherousset et al., 2012) est donc fortement relativisée. En effet, « sciences et techniques » n'est qu'une rubrique parmi quatorze. De plus, en ne réunissant que 14,4\% des articles, elle se place derrière « nature, environnement, écologie, planète » (21 \%) - ce qui n'est guère surprenant - et surtout derrière « insolite » $(23,6 \%$ et 3।,5\% si l'on ajoute « fun, buzz » d'inspiration voisine), ce qui l'est a priori davantage...

Les titres des articles constituent aussi un indice de l'altération ${ }^{16}$. La traduction française de l'article publié par PLOS ONE, « avalisée » par l'équipe de recherche, est: «"Les orques d'eau douce" : comportement d'échouage d'un poisson introduit afin de capturer des oiseaux terrestres $\gg .60 \%$ des articles sont plus ou moins en phase avec la deuxième partie du titre : ils mettent l'accent soit sur l'attaque (« Silures : des pigeons attaqués par des poissons »), soit sur la chasse («Quand les silures chassent les pigeons »; « Les silures ont trouvé de nouvelles proies »), soit sur la nourriture

\footnotetext{
15 Nous avons réuni dans une seule catégorie les rubriques appelées par les sites « environnement 》, « nature », « planète » ou « écologie » (quand il ne s'agit pas de la discipline scientifique) car leurs contenus sont souvent très proches.

${ }^{16}$ En n'utilisant pas le terme « silure » (« catfish ») pour désigner les poissons et en évoquant les « orques d'eau douce », la première partie du titre de l'article de PLOS ONE est déjà une altération susceptible d'en encourager d'autres.
} 
(«Des silures qui mangent des pigeons »), soit sur la nouveauté du comportement de l'animal («Évolution : les silures duTarn mangent des pigeons » ; « Quand un poisson se met à chasser le pigeon »). Seuls quelques-uns ont recours à un vocabulaire plus spécialisé : « Régime alimentaire surprenant du silure »; «Adaptation des techniques de chasse du silure »; « Évolution des mœurs du silure » ; « Des poissons ont modifié leur comportement en chassant des pigeons ».

Mais nombre de titres sont décalés, évoquant tour à tour (liste non exhaustive) :

- le repas (« À table, silures! »), les fines gueules (« Des silures amateurs de pigeons! »; « Les silures duTarn aiment les pigeons! »), la goinfrerie (« Quand les silures bouffent les pigeons! »-deux sites; « Le silure bouffe du pigeon! »; «Des silures qui sautent sur des pigeons et les dévorent! »)

- le combat (« Silures vs pigeons 》 - cinq sites);

- la fable de La Fontaine (Le loup et l'agneau) (« Le silure et le pigeon » - deux sites dont Wapiti, journal pour enfants) ;

- la dimension en partie locale de l'événement pour un site midi-pyrénéen (« Le Tarn fait le buzz sur le web ») ;

- l'impressionnant («Les silures attaquent les pigeons : images impressionnantes! » - trois sites), l'inquiétant (« Le silure, un poisson tueur de pigeons »; « Les silures, poissons redoutables, gobent les pigeons! »; « Dans le Tarn, les silures ne font qu'une bouchée des pigeons »), voire le terrifiant par l'évocation du requin (« Silures: ces requins d'eau douce ! »; « Le silure, requin d'eau douce, mangeur de pigeons! »; « Les silures requins des rivières »);

- la surprise mêlée de curiosité («Étonnants ces silures dans le Tarn ! » ; « Dans le Tarn, d'étonnants poissons se mettent à chasser le pigeon! » ; Une vidéo surprenante! ») et donc susceptible de faire le « buzz » (« Les silures chasseurs de pigeons font le buzz ! »- deux sites ; «Buzz : les silures mangeurs de pigeons » ; «Vidéo-buzz : les silures attaquent les pigeons »);

- l'appât humoristique du pêcheur (« Une bonne mouche pour les silures : le pigeon! »-site de pêche) ou du lecteur avec un titre sibyllin (« Silures vs pigeons : l'arroseur arrosé »).

Ces altérations ne sont pas nécessairement le fait des associations ou des nombreux médias natifs du web spécialisés dans le buzz, voire de la presse traditionnelle souvent soucieuse de faire de l'audience à travers une titraille racoleuse.Ainsi, par exemple, le titre de Sciences etAvenir évoque-t-il explicitement les « tueurs », quand celui d'Aquaportail (aquariophilie) parle d'adaptation des techniques de chasse, celui de Carnasse Pêche (association de pêcheurs) d'évolution des mœurs et celui de Nous ne sommes pas seuls (paranormal) de modification des comportements. 
Pour leur part, les commentaires des internautes relèvent de plusieurs registres, eux aussi non nécessairement en phase avec le site qui les publie ${ }^{17}$. Certains sont proches de l'esprit et/ou de la lettre de l'article initial : "Cette scène reste exceptionnelle car elle établit une connexion inédite entre les chaînes alimentaires et terrestres, un phénomène jusques là peu établi en eau douce » (Naturellement Pêche, Joss, 08/12/12). De tels propos auraient pu être tenus par un spécialiste de la vulgarisation ou par les chercheurs eux-mêmes. D'autres s'en éloignent pour mieux surexposer une dimension spécifique, sans pour autant trahir l'article de PLos ONE.Ainsi la prédation des espèces indigènes renvoie-t-elle à la biodiversité qui, elle-même, interroge la protection de l'environnement : « Peut-être ne fallait-il pas introduire ces poissons trop rapaces dans ces fleuves. lls ont dû trop vite dévorer les petits poissons et perturber leur cycle de reproduction » (Le Journal du Siècle, Magygaby, 08/I2/I2). Certains vont plus loin en dénonçant (ou en contestant) la nocivité du silure, voire de ceux qui les ont introduits ${ }^{18}:$ «Saletés de silures! Ca fait des années qu'ils sévissent en Moselle, tout ça pour que des vieux boucs puissent profiter de leur pêche "sportive" » (Réponse à Tout!, Anonyme, I |/12/12). Ou en demandant à une fédération de pêche ou à un maire (sic) de le faire interdire dans « nos » rivières et lacs. Plusieurs se situent dans le registre de l'humour, par exemple, en dénonçant la bêtise du pigeon : « C'est là que tu vois que c'est un con. II voit une grosse tache noire s'approcher, mais il continue à se mouiller le cul et puis il se fait péter le cul » (Divertissons nous, Damien, I / / 2//2). Ou encore en plaisantant sur le régime alimentaire du silure : « Un bon repas, quoi ! II n'y a pas de mal à se caler » (Maxisciences, Vito, 08/I2/12) ; « II est vrai que du poisson, toujours du poisson, ça devient monotone ! 》 (Maxisciences, Max 84300,08/I2/I2) ; « À quand les petits enfants ? » (BFMTV, Djtah, 12//2//2). Quand ce n'est pas en débordant sur celui de l'homme : « C'est pratique ces poissons! On en pêche un, et avec un peu de chance, il y a tout ce qu'il faut comme accompagnement dans son estomac! Plus qu'à trouver une poêle à frire assez grande » (Come4news, EricPomme, 12/12/12). Ou encore, en renvoyant de manière décalée à la littérature : «Après le succès de la fable Le corbeau et le renard, l'évolution du repas des renards vers le fromage est bien connue. Renardus souricus mute tranquillement en renardus camembertus (Sciences et Avenir, Daniel Pignard, 15//2//2).

Inévitablement, quelques-uns revisitent en l'enrichissant la thématique des dents de la mer : « Ça fait peur! Qu'en sera t-il si d'autres animaux mutent et attaquent les humains qui se baignent » (Le Journal du Siècle, Messager, 08/I2/I2) ; quelquefois ironiquement: «Tant qu'ils ne mangent pas les Albigeois... » (La Dépêche,Trabucaire,

\footnotetext{
17 II n'est pas rare que les auteurs des commentaires sur les sites de pêche ou dédiés aux animaux aient une plus grande proximité avec les chercheurs que ceux qui produisent des commentaires sur des sites scientifiques (voir infra).

${ }^{18}$ Quelquefois, les commentateurs des sites internet dénoncent les chercheurs : « Six chercheurs pendant des heures sur un pont et en 24 séances... Un beau métier! J'ai quand même l'impression que les pigeons ne sont pas seulement dans la vidéo » (Sciences et Avenir, Harry Vederci, I5/I2/ /2). Ou « la science » : « Des scientifiques l'ont filmé, alors c'est une réalité qui peut être admise par tous. Alors que les observations des pêcheurs... » (La Dépêche, Tier, 08/I2/I2).
} 
I ///2//2) ; « Un commentaire dit qu'un plongeur du Rhône fut englouti par un silure qui l'avait sûrement confondu avec un congénère. Je ne sais pas, mais en tout cas, ça génère pas mal de cons » (Esoxiste,Alban, 08/I2//2). D'autres transposent la voracité à d'autres champs : «Orange, SFR, Bouygues se sont attaqués pendant des années aux pigeons sans qu'aucun ne réagisse 》 (Maxisciences, Raudi, I0/12/I2) ; «Parmi nos politiques, certains doivent être croisés avec des silures vue leur voracité financière » (Midi Libre, JPP, 09//2/I2); « C'est loin d'être un scoop. Je peux même vous dire que les silures socialistes préviennent les pigeons avant de les manger et finissent toujours par abandonner leurs proies. Tandis que les silures de droite leur apportent des graines, s'inquiètent de leur santé et certains les aident même à construire leur nid » (Rue 89, Djambo, 08//2//2).

Jusque dans leur diversité, ces commentaires sont de même facture que ceux que l'on rencontre sur la plupart des forums, et ce quel que soit l'objet dont on discute : si certains le serrent de plus ou moins près, éventuellement en testant des entrées incongrues, d'autres s'en emparent pour évoquer ce qui leur tient le plus à cœur, pour parler d'eux et/ou rester dans l'entre-soi, car parler sur un forum ou un réseau social fait lien, et ce quel que soit le sujet et le type de protagonistes : parler politique en ligne (Réseaux, 2008), discuter entre supporteurs (Boure, 2013) ou entre adolescents sur leurs goûts musicaux (Laplante, à paraître).

Une fois encore, une polyphonie se dégage incontestablement et, qui plus est, une polyphonie étendue puisque tout est susceptible d'être mis en rapport avec tout, y compris avec n'importe quoi - au sens non péjoratif de l'expression par ces énonciateurs multiples et hétérogènes que sont les sites et les blogs, et au-delà par un public d'internautes encore plus large et hétérogène. Un fait scientifique est devenu un événement aux résonances multiples à travers une construction discursive polyphonique, mise en intrigue et en circulation par des acteurs individuels et collectifs multiples qui se répondent, s'opposent, se confortent, se détournent. Dès lors, il sort de « l'ordre des choses scientifiques » pour être propulsé dans d'autres sphères au sein desquelles il entre en résonance avec des enjeux, des intérêts, des valeurs, des représentations propres aux acteurs impliqués. Et cette polyphonie interpelle la science bien au-delà de la vulgarisation et de ses préoccupations stratégiques ou didactiques. Dans la mesure où elle modifie non seulement le rapport et les frontières entre le champ scientifique et d'autres champs - que celui-ci se plait à présenter comme extérieurs -, mais aussi les hiérarchies et catégories que les communautés scientifiques construisent, c'est aux représentations mêmes que la science (se) donne d'elle-même qu'elle s'attaque ${ }^{19}$. Finalement, à des degrés variables, ces acteurs s'autorisent à faire avec - au sens de Michel de Certeau - l'information scientifique proposée en pratiquant le détournement, le contournement, la surinterprétation, le braconnage.

\footnotetext{
${ }_{19}$ Ainsi que le souligne Johannes Angermüller (20 I I), les moteurs de recherche et autres agrégateurs accentuent ce phénomène en mélangeant, à l'aide d'algorithmes inconnus des utilisateurs, références scientifiques et références profanes.
} 
Cette pratique en rejoint une autre développée, par exemple, par lgor Babou et Joëlle Le Marec (2008) à partir d'une enquête sur les banques d'images d'institutions scientifiques françaises: les acteurs scientifiques ne sont plus seulement des producteurs d'énoncés éponymes, mais sont devenus des acteurs, et parfois des représentants « multicartes ». En effet, la sphère scientifique mobilise et agence de plus en plus ses ressources pour se tourner vers l'extérieur, et spécifiquement vers les médias et l'internet, afin de mettre en scène et en visibilité ses institutions, ses chercheurs et ses travaux... ce qui contribue à brouiller les frontières entre le scientifique et l'extrascientifique, les résultats de la recherche et leurs avatars et caricatures médiatiques et donc à inviter le profane à revisiter, à sa manière, la science. D'ailleurs, PLOS ONE, revue électronique en libre accès dont les articles sont sous licence Creative Commons - ce qui rend leur utilisation gratuite et ouverte (commentaires, annotations, évaluations, téléchargements...), y compris à des fins ludiques ou commerciales - est un exemple de ce phénomène. Même si son lectorat habituel est scientifique, elle est potentiellement consultable par un public plus large, voire très large en certaines circonstances, surtout depuis la création de Channel Plosone, accessible sur Youtube. On ajoutera que des mouvements de revendication portés par des chercheurs en faveur du libre accès, plus ou moins structurés mais de plus en plus visibles, tel celui qui a initié la pétition « I love open access » mise en ligne en mars $2013^{20}$, contribuent à amplifier ce phénomène.

\section{Dispositifs sociotechniques et lien social}

Puisqu'il est largement limité à la sphère internet, on peut penser que cet « emballement » est étroitement relié aux caractéristiques sociotechniques du web. En effet, ce dispositif fait une double promesse à tout producteur de messages ou d'informations : d'une part, la transmission quasiment instantanée à l'échelle mondiale, en première main ou en relais, de tout écrit, son, image fixe ou animée ; d'autre part, la démultiplication de la capacité mémorielle permettant à tout un chacun de consulter, mettre en perspective, vérifier. Encore faut-il que ces potentialités soient exploitées... ce qui est loin d'être le cas, tout message ou information ne donnant pas lieu, loin s'en faut, à une tornade numérique. Par ailleurs, Josiane Jouët (201 I :73) souligne que « La place du dispositif est cruciale dans la construction des usages sociaux des TIC [technologies de l'information et de la communication]. La culture numérique se ressource dans une dimension pragmatique qui articule le lien entre la matérialité des dispositifs et les modes de faire, la performativité des usages favorisant l'engagement dans de nouveaux régimes d'action ». Aussi faut-il s'interroger sur

\footnotetext{
20 « La connaissance ne saurait être traitée comme un bien classique et la circulation des savoirs est aujourd'hui plus que jamais un enjeu de société : il est possible de mettre en œuvre une révolution dans la démocratisation de l'accès aux résultats de la recherche. Un savoir enfermé derrière des barrières et accessible aux seuls happy few des universités les plus riches est un savoir stérile, et pour tout dire confisqué, alors qu'il est produit grâce à des financements publics »). Accès : http:// iloveopenaccess.org/open-access-manifesto/. Consulté le 03/I0/I 3.
} 
la participation, voire l'engagement des usagers, ainsi que sur leur capacité non seulement à maîtriser les modes opératoires et communicationnels, mais encore à intervenir sur le lien social et à gérer leur présence et leur visibilité sur l'internet. En conséquence, c'est bien vers l'articulation du social et du technique qu'il faut, une fois encore, s'orienter (Cardon, 2010 ; Lefebvre, à paraître).

\section{Les promesses de la vidéo}

La présence d'une vidéo attachée à l'article de PLOS ONE (Coucherousset et al., 20 I 2) a incontestablement contribué à la construction du processus d'amplificationaltération que nous venons d'expliciter. II faut se rappeler que se sont souvent des vidéos, spécifiquement quand elles sont mises en ligne sur Youtube, puis reprises et parodiées, qui sont à l'origine de « buzz » planétaires imprévisibles, démesurés et fascinants : Nyan cat - le chat arc en ciel qui rend fou -, Gangnam Style, Harlem Shake ${ }^{21} \ldots$ Dès lors, il est intéressant de vérifier si les caractéristiques de cette vidéo, vue plus de quatre millions de fois à l'échelle mondiale (voir supra) en quelques jours, permettent de comprendre sa circulation. Plus précisément, on se demandera si elle remplit les critères d'une vidéo formatée, consciemment ou non, pour circuler massivement sur le web, tout en sachant que la conformité à ces critères ne garantit nullement une diffusion élargie :

- la brièveté. La plupart des vidéos circulant massivement sur l'internet ne dépassent pas trois minutes et sont même plus courtes. Celle-ci dure 34 secondes. Cette caractéristique semble indiquer qu'une longueur excessive pollue l'attention et l'intérêt. D'ailleurs, cette remarque dépasse le web.Ainsi, pour capter et maintenir l'attention des téléspectateurs, la télévision propose-t-elle souvent des formats très courts (par exemple, la série Bref, Canal +, 20 I I-20I2) ;

- le décalage. II résulte de l'originalité de ce qui est montré, le jamais vu étant censé surprendre, voire provoquer un choc. Autrement formulé, ces images ont une forte indicialité : ce sont les seules représentations iconographiques scientifiques circulant sur l'internet du beaching des silures. Elles sont une trace qui atteste du caractère indiscutable du phénomène ;

- un sentiment de malaise. II est d'abord produit par la manière de filmer, comme si la réalité de l'image supplantait l'image de la réalité. Réalisé par les chercheurs, ce film entre dans la catégorie des « productions d'amateurs », omniprésentes sur le réseau car appréciées des internautes et facilement reconnaissables en raison de leur faible qualité technique compensée par le label d'authenticité qui leur est rapidement décerné. Par ailleurs, la manière de filmer est non seulement démonstrative, mais aussi dépouillée : succession rapide de plans fixes verticaux (la caméra est sur un pont), certains s'achevant sur un léger

\footnotetext{
21 À la mi-février 2013, la version originale de Gangnam Style a été vue près de I 500000000 de fois.
} 
zoom avant accompagnant l'attaque d'un silure, absence de commentaires au profit de sons naturels (bruit de l'eau qui coule, puis qui s'agite lors d'une attaque et quand le pigeon se débat)... D'ailleurs, on peut se demander si cette vidéo n'est pas « médiagénique » au sens défini par Philippe Marion (1997), autrement dit si elle n'est pas adaptée à l'internet en raison des spécificités du dispositif sociotechnique et sémiotique de la Toile. Aussi un malaise pourrait-il résulter de l'appel à l'imaginaire : d'abord, à l'imaginaire individuel - le cauchemar, manifestation onirique source d'anxiété, voire d'horreur ; ensuite, à l'imaginaire collectif à travers la réactivation de mythes et de croyances. En effet, dans de nombreuses mythologies, les éléments naturels (l'eau, l'air, la terre et le feu) jouent un rôle essentiel. Ils sont présents dans une multitude de récits dans lesquels ils sont incarnés par des personnages (ici les silures et les pigeons) vivant maintes péripéties et connaissant des fins contrastées. Sur un autre plan, on est en présence d'un phénomène qui interroge la modernité : la réactivation involontaire par la science de croyances qui, en repoussant les limites du concevable, renvoie aussi bien à des peurs (les monstres aquatiques, les mutants) qu'à des aspirations positives (dépasser son milieu naturel ou sa condition et se dépasser, repousser les interdits). Mais, en l'absence de véritables études qualitatives de réception, il ne s'agit que d'hypothèses ;

- le relais de la vidéo par des acteurs sociaux individuels et collectifs, influents ou anonymes, jouant le rôle de médiateurs à l'égard de publics larges ou spécialisés.

\section{Le jeu des médiations}

Quand une information mise en ligne est l'objet d'une circulation massive et accélérée, c'est d'abord vers la visibilité des nombreux acteurs collectifs et individuels qui l'ont relayée, puis vers l'intérêt matériel et/ou symbolique pour agir de ces acteurs, que l'on peut s'orienter pour comprendre pourquoi et comment cela se passe sur le plan de la circulation, mais également sur celui de la construction des multiples sens, décalés ou non par rapport aux sens proposés par les initiateurs.

\section{Une simple question de visibilité ?}

S'agissant d'une information scientifique, et même s'il est impossible à évaluer avec précision (voir supra), le nombre de médiateurs est élevé. Sont-ils pour autant visibles sur l'internet? Beaucoup de sites et blogs de notre corpus figurent en bonne place dans les classements du web français ${ }^{22}: 16$ se situent entre le

22 Le classement des sites internet français utilisé est celui d'UrImetriques (3//0 // 3). II est réalisé à partir d'un mode de calcul non explicité, mais qui prend en partie en compte le nombre de visites mensuelles et de pages mensuellement consultées. Nous l'avons comparé au classement établi le même jour par Alexa, dispositif dont les modes de calcul sont différents (décomptes 
premier et le $500^{\mathrm{e}}$ rang, sept entre le $50 \mathrm{l}^{\mathrm{e}}$ et le $\mathrm{I} 000^{\mathrm{e}}, 22$ entre le $\mathrm{I} 00 \mathrm{I}^{\mathrm{e}}$ et

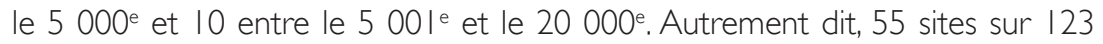
sont placés parmi les 20000 premiers dans un classement qui comporte plus de plus de 4500000 sites. Si ces sites sont largement consultés, les pages relatives aux attaques des silures ont-elles pour autant été visitées? On rappellera que, dès le lendemain de la publication de l'article de PLOS ONE, le blog « Passeurs de science » a été cité, pointé, repris et commenté par plus de la moitié des 123 sites et blogs de notre corpus (voir infra), ce qui lui confère une place spécifique dans le processus de circulation de l'information. Mais qu'en est-il des autres?

Avec les outils dont nous disposons, il est impossible de connaitre avec précision la consultation par les internautes des articles concernés et, a fortiori, le nombre de visiteurs uniques, sauf pour les rares sites qui affichent le décompte des visites de la page ou de la vidéo ${ }^{23}$. Ainsi, au 25 janvier 20।3, Rue 89 a reçu 84 I 59 visites, Réponse à Tout 7 468, Evilox 7 020, Le Dauphiné libéré 6 79I, Com4news 5 69I, Vidéo-buzz I 201, Buzzmoiça | 064, Aquagora 98I, Le Républicain lorrain 498 et Millau Live (site midi-pyrénéen) 308. Parmi les indicateurs possibles, figure aussi le nombre d'internautes qui ont recommandé l'article sur Facebook: parmi les plus actifs, on notera Passeur de sciences (7 200), Midi libre (I 700), La Dépêche (I 13I), Rue 89 (863), Pêcheur (725), Le Parisien (633), Le Journal du Siècle (577) Francetvinfo (499), Science et Avenir (405), Futura-Sciences (368), Radio Totem (36I), Maxisciences (34I), Le Point (294), Fun Buzz (256), Charente libre (162), Atlantico ( 158)... Si, à des degrés divers, certains de ces sites et blogs sont inscrits dans une logique d'audience (médias, notamment) et cherchent à atteindre de vastes auditoires, d'autres sont plus intéressés par la relation, la co-construction, l'interaction, le partage. Dès lors, la visibilité évaluée à la seule aune de l'audience n'est pas satisfaisante. Et quand recherche d'audience il y a, il convient de s'interroger sur ses causes. C'est-à-dire, qu'est ce qui peut pousser ces sites et blogs, hétérogènes du point de vue de leurs moyens, de leurs objets, de leurs modes de fonctionnement, de leur notoriété, etc. à relayer une information qui, a priori, semble peu susceptible d'attirer l'attention de publics larges? II est difficile d'imaginer qu'ils agissent sur le fondement de solidarités, ni même d'intérêts partagés et encore moins de façon concertée. C'est donc vers chaque catégorie d'acteurs qu'il faut chercher. Nous le ferons à travers les cas des médias et des collectifs de pêcheurs.

journaliers), mais tout aussi confidentiels. À l'exception de deux sites pour lesquels les écarts sont très importants (Bluzzin et Gobages, pour lesquels nous avons neutralisé le classement), les résultats s'inscrivent dans des ordres de grandeur voisins (par exemple, Urlmétriques place Canal + au $116^{\mathrm{e}}$ rang et Alexa au 14 $\left.\right|^{\mathrm{e}}$ ). Si les 5000 premiers sites sont dans une situation oligopolistique, les autres sont cependant largement consultés. Ainsi Actualités News environnement, classé 16128 par Urımétriques, reçoit-il plus de I 10000 visites mensuelles.

${ }^{23}$ La vidéo mise en ligne surYouTube par Pierre Barthélémy a ainsi été vue plus de 600000 fois (cf. supra). 


\section{Intérêt pour agir}

La socio-économie des médias l'a bien montré, les médias de l'internet sont intéressés par tout ce qui est susceptible de faire de l'audience en raison soit de la thématique et/ou du sujet, soit de la manière dont le sujet est traité. Cet objectif de maximisation de l'audience pour des raisons essentiellement économiques (Sonnac, 2009 ; Benghozi, Lyubareva, 20I2) ouvre en grand la porte au marketing du contenu et à ses pratiques sous-jacentes : sélection des informations et des angles de traitement en fonction des intérêts supposés des publics et des pratiques des internautes, habillage des articles (adjonction de photos, vidéos et de liens, titres accrocheurs, angles adaptés...). Simultanément, le référencement par Google (Rebillard, Smyrnaios, 2009) et, plus généralement, par les agrégateurs impose des normes de publication qui accentuent cette politique éditoriale. D'autant que nombre d'internautes accèdent aux articles moins en allant directement sur le site que par les liens proposés par les agrégateurs, voire par la personnalisation de l'accès à l'information fondée sur les flux RSs accessibles sur beaucoup d'appareils mobiles. Enfin, les médias ont fortement tendance à reprendre ce qui a du succès chez leurs concurrents, voire au-delà, la transhumance d'informations déclinée de l'intertextualité médiatique, devenant un mode normal de fonctionnement, voire une routine journalistique. Dit trivialement, le buzz suscite paresseusement le buzz, ce qui démultiplie le buzz. Parmi les sujets et les angles qui marchent et qui ont donc tendance à être privilégiés par les médias de l'internet, l'insolite occupe une place spécifique, surtout - comme c'est le cas ici - quand il est combiné à l'inquiétant, au spectaculaire (lié largement à la vidéo), au déconcertant, mais aussi à des images déjà vues, car très médiatisées, d'autres animaux pratiquant le beaching (orques, crocodiles...), au problématique du point de vue de l'environnement. L'insolite semble fonctionner comme une promesse d'intérêt pour les récepteurs.

II faut également prendre en compte la manière dont chaque média érige un fait en événement, ce qui renvoie sans doute à ses normes éditoriales, mais aussi à la capacité du fait à être mis en récit de manière à accrocher des publics élargis (Recherches en communication, 1997 ; Lits, 2008). Or, c'est bien de cela dont il s'agit dans notre cas : sous le regard des chercheurs et de leur caméra, des êtres réels (les silures et les pigeons) deviennent les personnages d'une histoire mise en scène, avec ses rebondissements, sa fin tragique (le pigeon est dévoré) ou heureuse (le pigeon échappe au prédateur), de sorte que les frontières s'estompent entre factuel et fictionnel, entre information, cognition et émotion, entre observation et imagination. En outre, on notera que le récit se construit non seulement dans chaque média, mais aussi dans plusieurs, à travers les références que les médias font à leurs homologues ainsi qu'à travers la circulation de flux amplifiée par la navigation intersites des internautes, par les réseaux sociaux, les flux RSs et les échanges de courriels.

Enfin, les médias doivent occuper leur propre espace, notamment avec des thématiques et des sujets récurrents qu'ils recyclent dans l'espoir de leur donner sinon une nouvelle vie, du moins un nouveau souffle. Or, pour les médias locaux imprimés ou en ligne, la pêche en général et la pêche aux grands carnassiers spécifiquement constituent de 
véritables marronniers de l'information. II suffit de consulter les archives d'un média local pour vérifier que le silure est régulièrement présent dans ses colonnes, photos et (plus rarement) vidéos à l'appui. L'information est souvent construite soit comme un événement local (concours de pêche dans un village, pêche record d'un silure...), soit comme l'enjeu d'un débat (pour ou contre le silure) dont les parties prenantes sont locales, mais dont l'enjeu est d'une toute autre dimension (biodiversité, par exemple). Généralement, ce sont des correspondants locaux (quand ils existent) qui réalisent ces sujets. Mais il peut arriver que des journalistes qui alimentant beaucoup les rubriques sciences ou environnement, voire qui écrivent occasionnellement ou non dans un magazine de pêche, soient sensibilisés à ces questions et produisent des articles de fond, et parfois de véritables dossiers ${ }^{24}$.

Le cas des sites et blogs de pêche, dont on rappellera qu'ils sont surreprésentés dans notre corpus, est différent. Ces associations peuvent être analysées comme des collectifs de pratiques (Flichy, 2008 ; Réseaux, 20 10) construits moins autour de la résolution de problèmes ou de la réalisation de projets que du partage d'intérêts, de passions, d'émotions, d'activités, de connaissances, mais aussi de l'expression de solidarités et de la construction de sociabilités. Leurs sites et leurs forums sont des lieux virtuels dans lesquels s'expriment autant le faire que l'être des adhérents. Ce sont des espaces communs de rencontres, d'expressions d'appartenance, d'échanges, de mise à disposition d'informations et de débats sur celles-ci, qui plus est, des espaces non uniques car les commentaires révèlent que certains membres se rencontrent, voire se fréquentent dans d'autres espaces virtuels (réseaux sociaux, courriels, SMs) et non virtuels (réseaux associatifs, concours de pêche...). Un regard panoramique et rétrospectif sur quelques sites (Art de la pêche, Gobages, Carnasse pêche, Club des saumoniers, Pêche en Seine, Powerticale, Predators fishing) montre que ces collectifs sont réactifs sur toute information, quelle que soit sa nature ou son origine, à partir du moment où elle a un rapport avec la pêche, les poissons et l'eau, et très réactifs lorsqu'elle questionne le noyau dur de cette activité ludique. Dès lors, il n'est rien d'étonnant à ce que magazines spécialisés, sites, blogs et participants aux forums repèrent, diffusent et commentent, quelquefois de façon experte, une information scientifique qui concerne d'abord un poisson carnassier, introduit - souvent clandestinement - dans les cours d'eau français par des pêcheurs amateurs de pêche sportive, dont la morphologie intrigue autant qu'elle attire ${ }^{25}$ et dont les comportements, très suivis par de nombreux pêcheurs ${ }^{26}$,

${ }^{24}$ Ainsi La Dépêche du Midi du 24 février 2013 (versions papier et en ligne) publie-t-elle un dossier d'une page (trois articles, trois encarts et sept photographies) sur le retour des poissons carnassiers.

25 La popularité du silure dépasse le monde de la pêche : quand on tape le mot « silure » sur Google images, on obtient environ 114000 résultats (25/0I/I3). Ce constat est aussi celui de Venere (Divertissons nous, I //I2/12) : « Les silures, ils vivent dans Google, plus précisément au niveau de Wikipedia, Youtube et Dailymotion ».

26 Les chercheurs toulousains (Coucherouset et al., 2012) confirment une information donnée sur plusieurs sites du corpus : ce sont des pêcheurs, avec lesquels ils ont des contacts anciens et plus ou moins réguliers, qui les ont avertis des modifications de comportement des silures à l'égard des pigeons (entretien). 
font débat au sein même des collectifs. Mais aussi une information relative à l'environnement lato sensu et à ses multiples problématiques adjacentes (de la pollution à la biodiversité, en passant par les écosystèmes).

Contrairement aux organisations de chasse, beaucoup d'associations de pêcheurs sont devenues non seulement très sensibles à ces thématiques - peut-être parce que toute atteinte grave au milieu aquatique met directement en danger le poisson et derrière lui la pêche -, mais encore actives sur le terrain, et ce bien au-delà du repeuplement des rivières : sensibilisation des adhérents aux questions environnementales, nettoyage des cours d'eau, actions de découverte de l'environnement aquatique auprès de publics diversifiés, interventions dans les écoles... Sans pour autant négliger le lobbying auprès des autorités nationales et locales, elles sont également engagées dans des pratiques d'expertise et des actions de partenariat avec des associations de défense de l'environnement ainsi qu'avec les pouvoirs publics. Déjà agréée comme des dizaines d'autres au titre des associations de protection de l'environnement, en 2012, la Fédération nationale de la pêche a signé une convention de partenariat avec le ministère de l'Éducation nationale pour l'éducation au développement durable. Dans leurs discours et leurs pratiques, surtout au niveau local (Brun, Pinet, 2002), beaucoup d'associations se sont démarquées, au moins partiellement, d'une posture relevée dans les années 90 par Paulette Lafaye et Laurent Thévenot (1993) : la convocation de la défense de la nature comme simple habillage pour justifier la défense d'intérêts corporatistes. À certains égards, nombre de leurs membres, parfois avec des arrière-pensées opportunistes, sont « non des porte-parole universels dotés d'une compétence généralisée, mais des personnes qui, grâce à la pratique d'un loisir ou à leur engagement sont dépositaires de savoirs et de savoir-faire techniques particuliers, souvent développés dans la proximité, et qui vont pouvoir parler au nom d'entités muettes comme les poissons et les rivières » (Gramaglia, 2008 : 135). De là à s'approprier l'information en y ajoutant un apport personnel, il n'y a qu'un pas... que certains franchissent d'autant plus aisément qu'ils pratiquent régulièrement ou occasionnellement le journalisme participatif comme « action par laquelle un citoyen ou un groupe de citoyens, joue un rôle actif dans le processus de collecte, de communication, d'analyse et de diffusion de nouvelles et d'informations » (Bowman, Willis, 2003 : 9).

\section{Expression, participation et appropriation}

Pascal Froissart (2007) invite à déconnecter partiellement la rumeur et le « buzz » de la dimension informationnelle en se tournant vers l'expressivité. L'idée est que le succès sur l'internet d'une production est aussi lié à la participation massive et (pro)active des internautes à travers non seulement le journalisme participatif, mais encore et surtout la mise en ligne de répliques parodiques, souvent humoristiques, qui modifient plus ou moins fortement la forme et le fond en proposant des mises en scène rappelant la scénarisation originaire, mais avec « quelque chose d'autre » ou dans lesquelles les auteurs se mettent eux-mêmes en scène de façon décalée, à travers un texte, une 
photo ou une vidéo. Il y a donc une véritable réappropriation par détournement, non pas tant pour créer que pour montrer sa connivence et la place que l'on accorde à l'entre-soi en participant à « une cérémonie sans enjeu » (ibid. : 84), mais susceptible de déboucher sur une surenchère de répliques autant diffuse que confuse. C'est donc bien autour de la question du lien social que le phénomène se construit.

Si l'article de Plos ONE (Coucherousset et al., 2012) et les vidéos de Youtube ne provoquent ni une production de répliques hilarantes, ni même une surproduction de répliques, ils suscitent toutefois de multiples réactions qui méritent d'être relevées et qui prennent la forme de mises en ligne (à leur tour susceptibles de circuler, y compris au-delà des aficionados de la pêche) :

- de films personnels montrant des scènes voisines avec d'autres animaux - un caneton, un caniche... - , voire avec un bébé (il s'agit d'un trucage) ou des liens avec des vidéos circulant sur l'internet, parfois depuis longtemps, et qui mettent en scène, avec ou sans trucage, d'autres attaques par beaching de prédateurs aquatiques, voire d'autres types d'attaques dont les pigeons sont victimes (par une tortue, un oiseau de proie, un chat). Aussi évoque et/ou commente-t-on des vidéos que l'on a vues, des histoires que l'on a entendues dans des conversations entre pairs (« Un collègue chasseur m'a parlé de silures qui attaquent les canards », Aquagora, Itarilda, 12/12/12).

- d'images fixes telles des photographies personnelles de pêcheurs de silures photographiés avec leurs prises, devenues trophées, des montages et photographies d'animaux autres que les silures et les pigeons (le 1 I/I2/l2, Baguera poste sur Animogen les photos de ses chats) ou encore des photographies trouvées sur l'internet (silure dans son élément naturel, silure mort, pigeon au sol ou en vol). Quelques contributeurs publient en ligne des dessins réalistes de silures, de pigeons ou de leurres de pigeons pour la pêche et, plus rarement, des dessins humoristiques ;

- de liens avec des articles ou des vidéos mis en ligne sur plusieurs sites (entrant principalement dans les catégories « pêche », « sciences », « insolite », « humour 》) relatifs au silure, parfois au pigeon, et qui peuvent aussi porter sur des questions non soulevées par les chercheurs toulousains (le silure est-il mangeable ?). Plus rarement, des internautes pointent vers d'autres produits ou lieux : la pétition d'un président d'association de pêche pour défendre le silure, un site commercial vendant des articles de pêche adaptés au silure ou un répulsif pour pigeons, la Convention de Berne protégeant le silure, le ministère des Finances pour que les pigeons ne se fassent pas plumer fiscalement par le Bercylure ;

- de commentaires construits comme des répliques d'articles de vulgarisation. Par exemple, ces deux très longs commentaires experts d'Alexdeparis ( $1 / / / 2 / 12)$, parus sur un site dédié à l'insolite et au buzz (2tout2rien.fr) et s'appuyant sur les travaux de plusieurs disciplines, citent de façon très précise certains d'entre eux et prennent position « scientifiquement » sur le silure (régime alimentaire, nocivité...) ; 
- d'idées pour des histoires que l'on a envie de (se) raconter ou qu'on laisse à d'autres le soin de développer : « Le poisson chat est un poisson inventé par les chats pour leur plan de domination de la terre » (Gamaniak, Jvoujvpas, I4/I2/I2), voire pour des écrits de science fiction : «Peu de personnes en France connaissent les Siluriens, une espèce extraterrestre de l'âge classique. C'est une race reptilienne » (Passeur de sciences, DrWho, 06/12/12).

On peut aller plus loin que Pascal Froissart et considérer que la participation au processus de circulation-altération peut aussi être une manifestation de la présentation de soi au sens d'Erving Goffman (1974) et, plus précisément, de la manière dont on entend se mettre en visibilité sur l'internet, surtout quand on est socialement peu audible. Par référence à Dominique Cardon (2010), on se trouverait alors dans le «Web clair-obscur » au sein duquel l'amateur plus ou moins éclairé et le contributeur ordinaire se confondent, le premier ne commentant plus une information parce qu'elle fait sens, mais parce qu'elle lui permet de mettre en scène sa vie, son opinion... Dès lors, la tentation est grande de mettre l'accent sur une dimension «personnelle » qui permettra de se démarquer. On en donnera deux exemples. D'abord la mise en avant, par le témoignage - « J'ai été témoin d'une scène approchante sur le Rhône en 1989 »(Passeur de sciences, Fnature, 06/12/12) -, l'évocation d'une expérience personnelle - « J'ai mangé du silure pêché sur le Rhône et je trouve ça bon » (Passeur de sciences, Fred, 06/12/12) -, l'exposition de ses goûts - « Passionné d'eaux vives et de salmonidés, j'aime particulièrement pêcher dans les ruisseaux à l'ultra-léger » (Naturellement Pêche, Jérôme, 08/12/12). Ensuite, l'adoption d'une posture particulière, celle de l'expert (en science, animaux, pêche...) plus ou moins distancié, par exemple en étalant des connaissances présentées comme avérées, ou du régulateur du débat- « Dommage. II manque beaucoup d'informations importantes dans ce journal, ce qui tempérerait certaines réactions des lecteurs » (Midi libre, Stef 34), « Un peu de calme, Petit Motard, tu sembles prendre les messages pour toi alors qu'ils ne te sont pas adressés (Humminbird, Fabien 14, I //12/12) - voire du donneur de leçons - « Vous êtes un doux rêveur. Le silure ne joue pas, il bouffe [...]. Quand les apprentis sorciers comprendront-ils qu'il faut arrêter de jouer avec le feu ? » (Le Journal du Siècle, Besse JP, |8/0|/|3), « Je ne veux pas vous faire la leçon, mais enfin... » (La Dépêche, Janjan 666, 07/I2/12).

\section{Conclusion}

Au terme de cet accompagnement distancié d'un article scientifique dans ses pérégrinations sur l'internet qui, usage après usage, de commentaire plus ou moins expert en retraitement ouvertement profane, l'ont conduit bien au-delà de la sphère scientifique, les interrogations restent nombreuses et appellent des investigations complémentaires. L'une d'elle est lancinante, en tout cas pour celles et ceux qui prennent la culture scientifique au sérieux : le processus de socialisation 
complexe et hétérogène des connaissances que nous venons de déconstruire à travers l'étude d'un cas (encore ?) peu ordinaire s'inscrit-il dans un processus plus large de démocratisation qui, in fine, placerait les acteurs individuels et collectifs du réseau au cœur d'un dispositif sociotechnique incitant à la participation ? Ou ne traduit-il pas plutôt le renforcement du rôle et de la légitimité des médiateurs, ce fameux « tiers » de la vulgarisation scientifique du siècle dernier? Mais d'un tiers singulièrement composite, polyphonique et inventif pour lequel la culture scientifique est une préoccupation très inégalement partagée et dont la production fait néanmoins culture, puisque les objets initialement construits par les chercheurs deviennent culturels du fait même de leur circulation créative (Jeanneret, 2008).

\section{Références}

Aldrin P., 2005, Sociologie politique des rumeurs, Paris, Presses universitaires de France.

Allport G., Postman L., 1947, The Psychology of Rumor, Oxford, H. Holt \& co.

Angermüller J., 201 I, « La science sur le web. Numérisation des textes et dédifférenciation du savoir », MEl, 35, pp. I27-। 40.

Babou I., 2004, Le cerveau vu par la télévision, Paris, Presses universitaires de France.

Babou Y., Le Marec J., 2008, « Les pratiques de communication professionnelle dans les institutions scientifiques. Processus d'autonomisation », Revue d'anthropologie des connaissances, I, vol. 2, pp. I I5- I 42.

Benghozi P.-J., Lyubareva I., 2012, Les modèles d'affaires des plateformes de contenus : étude et typologie à partir du cas de la presse, rapport, ministère de la Culture et de la Communication, déc.

Boure R., 2013, «Parler rugby en ligne entre soi. Conversations ordinaires sur le site Rugbyrama.fr », Réseaux, 180, pp. I57-187.

Bowman S, Willis C, 2003, We Media: How Audiences are shaping the Future of News and Information, Reston, American Press Institute. Accès : http://www.hypergene.net/ wemedia/download/we_media.pdf. Consulté le 12/03/I3.

Brun A., Pinet J.-M., 2002. Les pêcheurs et la qualité des milieux aquatiques : pour une approche locale des pratiques, Paris, Adeprina-CSP.

Cardon D., 20 I0, La démocratie internet. Promesses et limites, Paris, Éd. Le Seuil/La République des idées.

Charaudeau P., dir., 2008, La médiatisation de la science. Clonage, OGM, manipulations génétiques, Bruxelles/Paris, De Boeck/Ina.

Cheveigné S. de, 1997, « La science médiatisée : les contradictions des scientifiques 》, Hermès, 21 , pp. $121-134$.

Coucherousset J., Boulêtreau S., Azemar F., Compin A., Guillaume M., Santoul F., 2012, «"Freshwater KillerWhales": Beaching Behavior of an Alien Fish to Hunt Land Birds », PLOS ONE, 7 ( I 2). Accès : http://www.plosone.org/article/info\%3Adoi\%2FI0. I 37 I\%2Fjournal. pone.0050840. Consulté le 07//2/13. 
Degand A., Grevisse B., dirs., 2012, Journalisme en ligne. Pratiques et recherches, Bruxelles, De Boeck.

Fayard P., 1988, La communication scientifique publique. De la vulgarisation à la médiatisation, Lyon, Chronique sociale.

Flichy P., 2008, « Internet et le débat démocratique », Réseaux, | 50, pp. I59- 86.

Froissart P., 2002, La rumeur. Histoire et fantasmes, Paris, Éd. Belin.

— 2007, « Buzz, bouffées d'audience et rumeur sur Internet », Médiamorphoses, 21, pp. 8 I -87.

— 201 I, «Mesure et démesure de l'emballement médiatique. Réflexions sur l'expertise en milieu journalistique », MEl, 35, pp. | 4 | - 158.

Goffman E., 1974, La mise en scène de la vie quotidienne. La présentation de soi, t. I, Paris, Éd. de Minuit.

Gramaglia C., 2008, « Des poissons aux masses d'eau : les usages militants du droit pour faire entendre la parole d'êtres qui ne parlent pas », Politix, 83, pp. I33-I53.

Jacobi D., 1999, La communication scientifique : discours, figures, modèles, Grenoble, Presses universitaires de Grenoble.

Jeanneret Y., 2008, Penser la trivialité, vol. I, La vie triviale des êtres culturels, Paris, Hermès/ Lavoisier.

Jouët J., 201 I, « Des usages de la télématique aux Internet studies 》, pp. 45-82, in : Denouël J, Granjon F., Communiquer à l'ère numérique. Regards croisés sur la sociologie des usages, Paris, Presses des Mines.

Kaufmann A., 1993, "L'affaire de la mémoire de l'eau. Pour une sociologie de la communication scientifique », Réseaux, 58, pp. 67-89.

Lafaye P., Thévenot L., 1993, « Une justification écologique ? Conflits dans l'aménagement de la nature », Revue française de sociologie, XXXIV, pp. 495-524.

Laplante A., à paraître, « Exposer ses goûts musicaux sur Facebook : quelles conséquences sur l'e-réputation des adolescents », colloque E-réputation et traces numériques, Université Toulouse I Capitole.

Lefebvre M., à paraître, « Le web scientifique entre visibilité et accessibilité : la socialisation des débats scientifiques dans la sphère internet 》, colloque Enjeux et usages des TIC 201 I, Bruxelles.

Lits M., 2008, Du récit au récit médiatique, Bruxelles, De Boeck.

Marion P., 1997, « Narratologie médiatique et médiagénie des récits », Recherches en communication, 7, pp. 61-87.

Morin E. et al, 1969, La rumeur d'Orléans, Paris, Éd. Le Seuil.

Pailliart I., dir., 2005, La publicisation de la science, Grenoble, Presses universitaires de Grenoble.

Rebillard F., Smyrnaios N., 2009, « L'actualité selon Google. L'emprise du principal moteur de recherche sur l'information en ligne », Communication et Langages, I60, pp. 95- 109.

Recherches en communication, 7, 1997, « Le récit médiatique ».

Réseaux, 150, 2008, « Parler politique en ligne ».

— 164, 2010, « Les nouvelles formes des collectifs 》.

— 177, 2013, « Politique des algorithmes. Les métriques du Web ». 
Sonnac N., 2009, « L'économie de la presse : vers un nouveau modèle d'affaires », Les Cahiers du journalisme, 20, pp. 22-43.

Vatin F., 20I0, «L'incertitude du monde social et la raison métrologique : une approche pradmatique », Revue française de gestion, 203, pp. 164-171.

Veron, E., 1985, Les spectacles scientifiques télévisés. Figures de la production et de la réception, Paris, La Documentation française.

\section{Annexe}

\section{Corpus des sources internet utilisées (classées par ordre alphabétique des noms de domaine)}

20minutes.fr. Accès : http://mww.20minutes.fr/article// 061929/quand-poissons-chats-attaquent-pigeons/. Publié le II/I2//2.

24matins.fr. Accès : http://uwww.24matins.fr/silures-des-poissons-attaquent-et-mangent-les-pigeons-3065 I/. Publié le 08//2/12.

2tout2rien.fr. Accès : http://wwww.2tout2rien.fr/les-poissons-chats-chassent-les-pigeons-video/. Publié le $12 / 12 / 12$.

Actualites-news-environnement.com. Accès : http://www.actualites-news-environnement.com/29739Des-silures-mangent-pigeons.htm//. Publié le 12/12/12.

Actuzz.com.Accès : http://actuzz.com/video-des-silures-attaquent-des-pigeons-5368. Publié le I0/I2/I2.

Adrénergik. Accès : URL inconnue. Publié le 09/12/12.

Agoravox.tv. Accès : http://www.agoravox.tv/culture-loisirs/etonnant/article/au-bord-du-tarn-dessilures-3728 I/. Publié le | ///2/12.

Alvinet.com. Accès : http://www.alvinet.com/actualite/articles/dans-le-tarn-les-silures-attaquent-lespigeons-images-impressionnantes-15290466.html/. Publié le 07//2/l2.

Amisdesamis.com. Accès : http://unww.amisdesamis.com/t| | | 3-le-siluretueur-de-pigeons. Publié le 15/I2/12.

Amisdesbetes.info. Accès : http://www.amisdesbetes.info/article-etonnants-silures-chasseurs-depigeons-dans-le-tarn- I | 3273766.html/. Publié le 09//2/l2.

Animogen.com. Accès : http://mww.animogen.com/forums/viewtopic.php?f=7/ \&t=6350/. Publié le I I/I 2/ I2.

APML. Accès : URL inconnue. Publié le 20/0I//3.

Aqua49.com. Accès : http://www.aqua49.com/t| 8 /66-silure-vs-pigeo. Publié le 06/12//2.

Aquaportail.com. Accès : mww.aquaportail.com/topic-7734-I4-adaptation-des-techniques-de-chasse-du -silure.html. Publié le 10/12/12.

Art-de-la-peche.com. Accès : http://www.art-de-la-peche.com/t38|74-quand-des-silures-attaquentet-mangent-des-pigeons. Publié le I // /2//2.

Atlantico.fr. Accès : http://www.atlantico.fr/atlantico-light/quand-poissons-albi-attaquent-et-mangentpigeons-5734 | 2.html/. Publié le I I/I2/ /2.

Bfmtv.com. Accès : http://www.bfmtv.com/planete/pigeons-attaques-silures-tarn-402912.html/. Publié le $12 / 12 / 12$. 
Blogbang.com. Accès : http://www.blogbang.com/articles/4079354-dans-le-tarn-les-silures-attaquentles-pigeons-images-impressionnantes.html. Publié le 07/12/12.

Blogwapiti.com. Accès : http://blogwapiti.com/actus/le-silure-et-le-pigeon/. Publié le 12//2//2.

Bluzzin.com. Accès : http://bluzzin.com/article/silures-attaquent-mangent-pigeons-72892.html. Publié le $1|/| 2 / 12$.

Buzzmoiça.fr. Accès : www.buzzmoica.fr/video/des-poissons-mangent-des-oiseaux-36907»»

Canalplus.fr.Accès : http://mww.canalplus.fr/c-infos-documentaires/pid2800-c-le-jt-de-canal.html?vid=775700/. Publié le 08/12/12.

Carevox.fr. Accès : http://www.carevox.fr/sante-des-animaux/article/des-pigeons-attaques-par-des/. Publié le $08 / 12 / 12$.

Carnapesac38.skyrock.com. Accès : http://carnapesca38.skyrock.com/3 I 29826226-Silure-VS-Pigeons. html. Publié le 08//2/l2.

Carnasse pêche. Accès : URL inconnue. Publié le 12/12/12.

Carpealsace.com. Accès : http://www.carpealsace.com/t I5839-silures-attaquent-des-pigeons. Publié le $10 / 12 / 12$.

Carredinfo.fr. Accès : http://carredinfo.fr/revue-du-web-de-la-semaine- | 4-chauffeur-braqueur-poissonscolombophages-et-bambous-de-sauvetage- 17487/. Publié le 09/12/12.

Charentelibre.fr.Accès : http://www.charentelibre.fr/20 I 2/12/07/quand-les-silures-se-mettent-a-chasserles-pigeons-video, I I 28680.php/. Publié le 07//2//2.

Chassepassion.net. Accès : http://www.chassepassion.net/le-forum/35/25864. Publié le I I/I2/I2.

Chassimages.com. Accès : http://wnww.chassimages.com/forum/index.php?topic=1 71 365.20;wap2. Publié le $09 / 12 / 12$.

Clubcarna77.forumactif.org. Accès : http://clubcarna77.forumactif.org/t3204-quand-les-silureschassent-et-mangent-les-pigeons. Publié le 08//2/12.

Cnrs.fr. Accès : http://www.cnrs.fr/inee/communication/breves/frederic_santoul20I2.htm/. Publié le $06 / 12 / 12$

Come4news.com. Accès : http://www.come4news.com/les-poissons-mangeurs-de-pigeons-442568/. Publié le I|//2/12.

Directmatin.fr. Accès : http://wnww.directmatin.fr/environnement/20 12-12-07/video-des-pigeons-attaquespar-des-poissons-275505/. Publié le 07/12/12.

Divertissonsnous.com. Accès : http://www.divertissonsnous.com/20 1 2//2// I/un-poisson-chat-attaquedes-pigeons/. Publié le I ///2//2.

Esoxiste.com. Accès : http://esoxiste.com/video-les-silures-mangeurs-de-pigeons/. Publié le 09//2/l 2.

Exo-thonic.purforum.com,. Accès : http://exo-thonic.purforum.com/t I 5 | 3-les-silures-et-les-pigeonsa-albi-tarn. Publié le I///2/12.

Federationdesacteursruraux.blogspot.fr. Accès : http://federationdesacteursruraux.blogspot.fr/20 I 2//2/ des-silures-attaquent-et-mangent-des.html. Publié le 09//2//2.

Fluctuat.premiere.fr. Accès : http://fluctuat.premiere.fr/Societe/News/Evolution-les-silures-du-Tarn-mangentdes-pigeons-3596842/. Publié le 12/12/12.

Forum.aquagora.fr.Accès : http://forum.aquagora.fr/2 I/silures-mangeurs-de-pigeons/20/.Publié le I2/12//2.

Forum.club-des-saumoniers.org. Accès : http://forum.club-des-saumoniers.org/viewtopic.php?id= 693. Publié le 08/12/12.

Forum.predators-fishing.com. Accès : http://forum.predators-fishing.com/viewtopic.php?id=|3819. Publié le $07 / 12 / 12$ 
Forumbretagne-vivante. Accès : http://www.forumbretagne-vivante.org/t5857-pigeon-predates-parlesilure. Publié le 07/12/12.

Forums.bluebelton.com. Accès : http://forums.bluebelton.com/nos-loisirs-nature-f39/quand-les-siluresbouffent-les-pigeons-t 19883.htm. Publié le 08/I2/I2.

Forums.france2.fr. Accès : http://forums.france2.fr/france2/jtfrance2/silures-pigeons-sujet_56I7I_l. htm. Publié le 10/12/12.

Forumter.com. Accès : http://www.forumter.com/t9389-pigeons-vs-silures. Publié le 08//2//2.

Fr.evilox.com. Accès : http://fr.evilox.com/videos/silure-vs-pigeon. Publié le 19//2/12.

Franceinter.fr. Accès : http://www.franceinter.fr/reecouter-diffusions/2446 |7/. Publié le I0/I2/I2.

Francetvinfo.fr. Accès : http://www.francetvinfo.fr/video-les-silures-d-albi-ont-trouve-de-nouvellesproies-des-pigeons_184607.html/. Publié le 10/I2/12.

Frituremag.info. Accès : http://www.frituremag.info/Home-Page/Video-inedite-dans-le-Tarn-les.html/. Publié le 10/12/12.

Funbuzz.com. Accès : http://wnww.fun-buzz.com/. Publié le | ///2//2.

Fun-buzz.com. Accès : http://www.fun-buzz.com/2012//2// //des-silures-qui-attaquent-et-mangentdes-pigeons/. Publié le | ///2//2.

Futura-sciences.com. Accès : http://wnww.futura-sciences.com/fr/news/t/zoologie/d/en-video-des-poissonschats-albigeois-se-nourrissent-de-pigeons_43307/. Publié le I0//2/12.

Gamaniak.com.Accès : http://www.gamaniak.com/video-8768-poisson-chat-attrape-pigeon.html/. Publié le $11 / 12 / 12$.

Gobages.com. Accès : http://www.gobages.com/forum-mouche/showthread.php?t=37762. Publié le $06 / 12 / 12$

Gurumed.org. Accès : http://www.gurumed.org/2012/I2/08/quand-les-poissons-chats-sattaquent-auxpigeons-urbains-vido/. Publié le 08//2/l2.

Hellocoton.fr. Accès : http://www.hellocoton.fr/to/sBSo\#http://www.terrafemina.com/vie-privee/sante/ articles/20427-attaque-de-pigeons-par-des-silures-video.html/. Publié le 10/12/12.

Hisse-et-oh.com. Accès : www.hisse-et-oh.com/forums/la-taverne/messages/I 230706-silures-a-l-attaquede-pigeons. Publié le 09//2/12.

Humminbird.xooit.fr. Accès : http://humminbird.xooit.fr/t4488-Etonnant-ces-silures-dans-le-TARN. htm. Publié le 09/12/12.

Hydrobioblog.blogvie.com. Accès : http://hydrobioloblog.blogvie.com/2012/12/29/regime-alimentairesurprenant-du-silure/. Publié le 29//2/12.

Infos-chalon.com. Accès : http://www.infos-chalon.com/article.php?sid=38585\#.UMruWmazgkg/. Publié le $08 / 12 / 12$.

Jack35.wordpress.com. Accès : http://jack35.wordpress.com/20 I2/I2/08/dans-le-tarn-detonnants-poissonsse-mettent-a-chasser-le-pigeon-video/pigeon-et-poisson/. Publié le 08/I2/l2.

journaldelascience.fr. Accès : http://www.journaldelascience.fr/search/node/silures\%20et\%20pigeons. Publié le 08/12/12.

Koreus.com. Accès : http://www.koreus.com/modules/newbb/topic90 I 20.html. Date inconnue.

Ladepeche.fr. Accès : http://www.ladepeche.fr/article/20 I 2/I 2/07// 508520-albi-quand-les-silures-dutarn-mangent-des-pigeons.html/. Publié le 07//2/12.

Ladepeche.fr. Accès : http://www.ladepeche.fr/article/20 I 2/I 2/08/I 509233-albi-les-silures-chasseursde-pigeons-font-le-buzz.html/. Publié le 08//2/l2. 
Ladepeche.fr. Accès : http://www.ladepeche.fr/article/20 | 3/0 I/08//53 I0 /4-albi-marc-et-antoinetteont-filme-les-silures-chasseurs-de-pigeons.html. Publié le I //0 ///2.

LCI. Accès : http://blog-lci-est-a-vous.lci.fr/page/3 I. Publié le I ///2/12.

Ledauphine.com. Accès : http://mww.ledauphine.com/france-monde/20 I2//2/08/des-silures-se-nourissantde-pigeons/. Publié le 08//2/12.

Lejournaldusiecle.com. Accès : http://lejournaldusiecle.com/20 / 2/ /2/08/quand-des-poissons-attaquentet-mangent-des-pigeons/. Publié le 08/I2/I2.

Leparisien.fr. Accès : http://www.leparisien.fr/environnement/video-albi-quand-les-silures-chassent-despigeons-08-12-20 I2-239 / 033.php. Publié le 08/I2/l2.

Leparisien.fr. Accès : http://www.leparisien.fr/toulouse-31000/les-silures-poissons-redoutables-gobentles-pigeons-du-tarn-07-12-20 /2-23887/7.php. Publié le 07//2/12.

Lepoint.fr. Accès : http://www.lepoint.fr/science/qui-sont-ces-poissons-qui-gobent-les-pigeons-dutarn-07-12-2012-1547082_25.php. Publié le 07/12/12.

Lesamisdedidie.net. Accès : www.lesamisdedidie.net > ... > Univers marin. Publié le 08//2/I2.

Lesmotsontunsens.com. Accès : http://mww.lesmotsontunsens.com/video-les-silures-chasseurs-de-pigeonsfont-le-buzz-136/5. Publié le I0/I2/I2.

Lespluslus.blogspot.fr. Accès : http://lespluslus.blogspot.fr/20 I2//2/des-silures-attaquent-et-mangentdes.html. Publié le 09//2/12.

lespostiers.fr. Accès : http://lespostiers.fr/2012/12/10/quand-le-pigeon-se-fait-chasser-par-des-poissons/. Publié le I0//2/12.

Lindependant.fr. Accès : http://www.lindependant.fr/20 I / / 2/ I 0/tarn-quand-les-silures-attaquent-lespigeons, I 709762.php/. Publié le 10//2//2.

Littlefishing.overblog.com. Accès : http://littlefishing.overblog.com/silure-vs-pigeons»》

Maxisciences.com. Accès : www.maxisciences.com > Planète > Poisson > News. Publié le 07//2/l2.

Mcetv.fr. Accès : http://mcetv.fr/news-express/ I I 2-des-poissons-chats-attaquent-et-devorent-despigeons-video. Publié le | ///2/12.

Midilibre.fr. Accès : http://www.midilibre.fr/20 I 2/12/08/des-silures-attaquent-et-mangent-des-pigeonssur-le-berges-du-tarn,60826 I.php/. Publié le 08//2//2.

Midi-pyrenees.france3.fr. Accès : http://midi-pyrenees.france3.fr/20 I 2/12/07/en-video-dans-le-tarnles-silures-se-nourrissent-de-pigeons-159947.html/. Publié le 07//2/12.

Millaulive.fr. Accès : http://wnww.millau-live.fr/videos/44-yannick-perie/video/56-dans-le-tarn-des-poissonsattaquent-des-pigeons.html/. Publié le 06/I2//2.

Mortderire.com. Accès : http://www.mortderire.com/actualites-insolites.html. Publié le I I/I2/I2.

Myreplay.tv. Accès : http://myreplay.tv/v/OOlef8SS. Publié le 07//2/12.

Natureetpassions.com. Accès : http://www.natureetpassions.com/t l 66I-Silures-et-pigeons-dans-leTarn.htm. Publié le 07//2/12.

Naturellement-peche.info. Accès : www.naturellement-peche.info/goodies/le-silure-requin-deau-douce? Publié le 08//2/12.

Nautictube.com. Accès : http://www.nautictube.com/incroyable-des-silures-attaquent-et-mangentdes-pigeons/. Publié le 09//2//2.

Nbekblog.free.fr. Accès : http://nbekblog.free.fr/?p=2035. Publié le 07//2//2.

Newsyahoo.com.Accès: http://fr.news.yahoo.com/poissons-dalbi-attaquent-mangent-pigeons-07/524043. html/. Publié le | I//2/12. 
Nousnesommespasseuls.xooit.com.Accès : http://nousnesommespasseuls.xooit.com/t l 8445-Des-poisssonsont-modifie-leur-comportement-en-chassant-des-pigeons.htm?q=silure+pigeon. Publié le 09//2/l 2.

Over-blog.com. Accès : ttp://www.over-blog.com/recherche/recherche-blog.php?ref=|4|837\&query =silures+et+pigeons. Publié le 10/12/12.

Passeurdesciences.blog.lemonde.fr. Accès : http://passeurdesciences.blog.lemonde.fr/2012//2/06/ dans-le-tarn-detonnants-poissons-se-mettent-a-chasser-le-pigeon/. Publié le 06/I2/l2.

Pecheenseine.com. Accès : wmw.pecheenseine.com/t2370 I-quand-les-silures-chassentles-pigeons. Publié le $08 / 12 / 12$.

Pechemaster.com. Accès : http://mww.pechemaster.com/videos/buzz/les-silures-attaquent-les-pigeonsdans-le-tarn- I I.html. Publié le 28//2/12.

Peche-poissons.com. Accès : http://www.peche-poissons.com/les-news/buzz-les-silures-mangeursde-pigeons/. Publié le 12/12/12.

Pêcheur.info. Accès : http://pecheur.info/silure-attaque-pigeon.html. Publié le 07//2/12.

Pecheursdu24.forumactif.org. Accès : http://pecheursdu24.forumactif.org/t I 0-des-silures-attaquentdes-pigeons. Publié le 07/0 I/13.

Phil3665.blogspot.fr.Accès : http://phil3665.blogspot.fr/20 I 2//2/le-silure-et-les-pigeons.html. Publié le $07 / 12 / 12$.

Pigeon-voyageur.com. Accès : http://pigeon-voyageur.com/index.php?mod=forum\&ac=voir\&id=4267. Publié le 08//2/12.

Playtv.fr. Accès : http://playtv.fr/video/246410/silures-des-pigeons-attaques-par-des-poissons-toulouse/. Publié le $07 / 12 / 12$.

Powerticale.forumpro.fr. Accès : http://powerticale.forumpro.fr/t3253-les-silures-attaquent-les-pigeons. Publié le 17/12/12.

Pseudonyme.over-blog.net. Accès : http://pseudonyme.over-blog.net/article-veille-quand-je-penseque-certains-ont-peur-de-se-baigner-dans-la-mer-a-cause-des-requins-il-y- I | 3368768.html. Publié le $15 / 12 / 12$.

Radio-totem.net. Accès : http://www.radio-totem.net/actualite/journal/all/article-6569 I-albi-des-siluresamateurs-de-pigeons/?page=\&filtre=all/. Publié le 07/12/12.

Reponseatout.com.Accès : http://mww.reponseatout.com/insolite/images-insolite/des-poissons-mangeurspigeons-a |08892/. Publié le I //I2/12.

Republicain-lorrain.fr. Accès : http://www.republicain-lorrain.fr/actualite/20 I 2/12/08/les-silures-du-tarngobent-les-pigeons/. Publié le 08//2/12.

Reviewer.fr. Accès : http://www.reviewer.fr/breves/web-tech/l 8402/quand-pigeons-font-bouffer-parsilures-video.html/. Publié le 10//2//2.

Rondbleu.com. Accès : mww.rondbleu.com/.../8995-de-poissons-carnassiers-mangent-des-pigeo...?. Publié le $11 / 12 / 12$.

Rue89.com. Accès : http://www.rue89.com/zapnet/20 I 2/12/09/des-silures-attaquent-des-pigeons-unevideo-scientifique-et-gore-237659/. Publié le 09/12/12.

Sciencesetavenir.nouvelobs.com. Accès : http://sciencesetavenirnouvelobs.com/nature-environnement/20121214. OBS2537/le-silure-poisson-tueur-de-pigeons.html/. Publié le 14//2/12.

Session-peche.conceptforum.net. Accès : http://session-peche.conceptforum.net/t5 | 4-silures-specialisesdans-la-chasse-aux-pigeons/. Publié le 08/I2/I2.

Silurusglanis.free.fr. Accès : http://silurusglanis.free.fr/bb3/viewtopic.php?f=34\&t= | 9239. Publié le 09/| 2// 2. 
Slate.fr. Accès : http://blog.slate.fr/globule-et-telescope/20 /2//2/l0/dans-le-tarn-des-poissons-chatspratiquent-la-chasse-aux-pigeons/. Publié le 10/12/12.

Sur-la-toile.com. Accès : http://www.sur-la-toile.com/article-1 17236-Remarquable-phenomene-d-unpoisson-chat-chassant-le-pigeon.html/. Publié le 08//2//2.

Tachka.jura-web.fr. Accès : http://tachka.jura-web.fr/?tag=silures-pigeons-tarn-attaques/. Publié le 08//2//2.

Tarulympique.com. Accès : http://www.tarulympique.com/article-dans-le-tarn-des-silures-attaquentdes-pigeons-I | 33598 | 3.html. Publié le I |/I2/I2.

Techno-science.net. Accès : http://www.techno-science.net/?onglet=news\&news=1। I09. Publié le 07/12/12.

Terrafemina.com. Accès : http://wnw.terrafemina.com/vie-privee/sante/articles/20427-attaque-de-pigeonspar-des-silures-video.html. Publié le I0//2//2.

Toppeo.com. Accès : http://www.toppeo.com/video-des-poissons-chats-attaquent-des-pigeons- 8968. html. Publié le 08/I2/12.

Toulouse7.com. Accès : http://www.toulouse7.com/20 I 2/ / 2/07/dans-le-tarn-les-silures-attaquent-lespigeons-images-impressionnantes/. Publié le 07//2/12.

Toute-l'actu.com,. Accès : http://toute-lactu.com/20 /2/12/I I/dans-le-tarn-les-silures-ne-font-quunebouchee-des-pigeons/. Publié le I ///2/12.

Univ-tlse3.fr. Accès : http://wwww.univ-tlse3.fr/077/86/2/0/fiche__actualite/\&RH=ACCUEIL. Publié le $06 / 12 / 12$.

Video-buzz.eu. Accès : http://video-buzz.eu/des-poissons-chats-attaquent-des-pigeons?clearCache=ouil. Publié le 08//2/12.

Web-tech.fr. Accès : http://web-tech.fr/des-silures-qui-sautent-sur-des-pigeons-et-les-devorent/. Publié le $13 / 12 / 12$.

WikiStrike.com. Accès : http://www.wikistrike.com/article-dans-le-tarn-des-poissons-chassent-des-pigeons| | 3247208-comments.html/. Publié le 08//2/12.

Youlab.fr. Accès : http://www.youlab.fr/blog/poisson-silure-pigeons/. Publié le 15//2//2. 